\title{
Optimization of pilot overhead in communications with ARQ-feedback
}

\author{
Besma Smida, Maliha Hossain and Yi Zhao
}

\begin{abstract}
In this work, we optimize the pilot overhead in communication with re-transmission and investigate the dependence of this overhead on various system parameters, e.g. fading rate, target rate and signal-to-noise ratio. We formalize the optimization and frame it in terms of throughput maximization; this leads to an accurate optimal pilot overhead closed-form expression. Results show that the optimal overhead expressions are roots of quartic polynomials. At a high-power regime they decrease with the target rate and increase with the normalized Doppler frequency, and at low-power regime they are only function of the target rate.
\end{abstract}

\section{INTRODUCTION}

Wireless networks must significantly improve their information rate in order to meet the increasing demand for highspeed data services. One key limit to the information rate is low packet efficiency: a packet typically carries less than $60 \%$ to $70 \%$ of actual data. The goal of this research is to increase packet efficiency by optimizing the pilot overhead. In this paper, we consider the common scenario of communication with ARQ feedback. Our pilot-overhead optimization take into account the tradeoff between training and re-transmission. Allocating more resources to training reduces the number of re-transmissions needed for successful decoding, so the system performance may therefore be dominated by the channel estimation accuracy. In contrast, by allocating fewer resources to training, the number of re-transmissions may increase and the performance may be dominated by the number of re-transmissions. To investigate this tradeoff, we used the throughput formulation - not the ergodic capacity formulation - derived in our previous paper [1]. This throughput formulation led to a numerical computation of the optimal pilot overhead. This paper expands upon the original numerical analysis [1] by analytically optimizing the pilot overhead in the case of ARQ-feedback. This leads to a quantification of the dependence of this overhead on various system parameters.

\section{A. Related works - Pilot overhead optimization}

The optimization of pilot overhead, predicated on the maximization of the ergodic channel capacity, has been largely studied in the literature [2]-[11]. In more common systems, where the pilot symbol power is fixed, the optimization is over the number of pilot symbols. In that case, some explicit results have been established in both low and high power regimes. Numerical solutions are derived for general power levels. By maximizing a tight lower-bound of the average channel capacity, a closed-form solution for the average rate of pilot symbol in block-fading [5] and in continuous fading with rectangular Doppler spectrum is derived in [7]. More recently, the optimization of the pilot overhead in a unified continuous and block-fading model is investigated in [12], [13], and the dependence of the optimum overhead on various system parameters of interest (e.g., fading rate, signal-to-noise ratio) is quantified. We studied training in two-way communication with re-transmission and derived an expression for throughput that takes into account training bits [1]. This paper is an extension of this work - it provides an analytical optimization of the pilot overhead in the case of ARQ-feedback.

Remark. All current wireless systems include Automatic Repeat reQuest (ARQ) protocol ${ }^{1}$ to ensure reliable delivery of data. Therefore, we decided to employ a framework that takes into account re-transmission when optimizing the pilot overhead. This throughput formulation will lead to more practical results. Indeed, the prior works - posed on maximization of the ergodic channel capacity - showed that one would select the number of pilot symbols to be as small as possible assuming that the SNR is sufficiently high [5], [8], [9]. This small number can be justified thus: the resources allocated to training directly reduce the amount of data in the forward channel, reducing the rates in the pre-log factor outside the $\log (1+$ SNR) (SNR is Signal to Noise Ratio) of capacity expressions for Gaussian channels. However, SNR-gain due to training improves rates inside the log. With our throughput formulation [1], the SNR-gain due to training not only improves rates inside the $\log$ but also reduces the number of re-transmissions, which leads to more realistic tradeoffs.

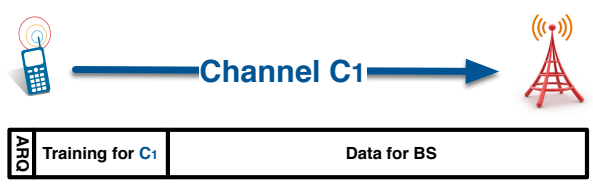

Fig. 1: The packet structure.

\section{B. Contributions}

The key departure from prior work on pilot overhead optimization is that we (a) consider a continuous time fading model that is more general than block Rayleigh, (b) find an accurate analytical expression for the optimal number of pilot symbols for communication with ARQ feedback, and (c) investigate the dependence of this overhead on various

\footnotetext{
${ }^{1}$ ARQ scheme may be improved by various packet combining schemes that may be loosely arranged into two categories: diversity-combining and incremental redundancy.
} 
system parameters, e.g. fading rate, target rate and signal-tonoise ratio.

\section{PRELIMINARY}

We consider a point-to-point communication with ARQ feedback as illustrated by Fig. 1, in which one end-user wishes to send messages to a central node, or base-station ${ }^{2}$. We assume that the receiver sends a ACK or NAK to indicate the success/failure of transmission ${ }^{3}$. The transmitter moves on to the next message in the transmission queue if it receives an ACK and re-transmits if it receives a NAK. In this work, we consider basic-ARQ; the transmitter sends the same packet and the receiver discards the erroneous packets.

\section{A. Channel Model}

In this system, we consider a Rayleigh fading channel and additive white Gaussian noise (AWGN). Let each packet contain $T$ symbols. The transmission $s$ is divided into two phases: training $\left(T_{t}(s)\right.$ symbols) and data transmission $\left(T-T_{t}(s)\right.$ symbols). Under this model, the input-output relationship of $i^{\text {th }}$ received symbol in the $s$-th packet is given by:

$$
y_{s}(i)=h_{s}(i) x_{s}(i)+w_{s}(i), i=0,1, \ldots, T-1
$$

where $x_{s}(i)$ is the $i^{\text {th }}$ symbol in the $s^{\text {th }}$ transmitted packet ${ }^{4}$ of constant power $P, y_{s}(i)$ is the corresponding received symbol, $w_{s}(i)$ is AWGN with zero mean and variance $N_{0}$ and the channel gain $h_{s}(i)$ is a discrete-time complex Gaussian stationary random process, with a continuous spectral distribution function whose derivative is the Doppler spectrum [13], [14].

\section{B. Channel Estimation}

The $T_{t}(s)$ training symbols are used to estimate $h_{s}(i)$ for all $i$ in the data transmission phase ${ }^{5}$. We evaluate the minimum channel estimation error of the channel vector $\mathbf{h}_{s}:=$ $\left[h_{s}(0), \ldots, h_{s}(T)\right]^{T}$, as a function of $T_{t}(s)$, which is needed to subsequently derive the throughput. Without loss of generality, we normalize the Rayleigh fading channel $\left(\left|\mathbf{h}_{s}\right|^{2}=1\right)$. Let $\widetilde{\mathbf{h}}_{s}=\mathbf{h}_{s}-\widehat{\mathbf{h}}_{s}$ denote the mismatch between the true channel $\mathbf{h}_{s}$ and its estimate $\widehat{\mathbf{h}}_{s}$. Assuming Minimum Mean Square Error (MMSE) estimation, we get the estimation error of the channel vector $\mathbf{h}_{s}$ at the receiver side as:

$$
\sigma_{\widetilde{\mathbf{h}}}^{2}(s)=\frac{1}{1+\frac{T_{t}(s) P}{2 f_{D} T N_{0}}}
$$

for a rectangular Jake's power spectrum [7], [13], where $f_{D} \leq 0.5$ is the Doppler frequency normalized by the symbol rate. Based on the orthogonality principle of the MMSE estimate, the channel estimation and the estimation error are uncorrelated. The random variables $\left|\widetilde{\mathbf{h}}_{s}\right|^{2}$ and $\left|\widehat{\mathbf{h}}_{s}\right|^{2}$, derived from uncorrelated complex Gaussian, are independent and

\footnotetext{
${ }^{2}$ This study is valid for frequency-division duplexing (FDD) and timedivision duplexing (TDD).

${ }^{3}$ Note that to increase the feedback reliability, the feedback ACK/NAK data may be coded over a larger block length.

${ }^{4} \mathrm{We}$ assume that the pilot and data symbols have the same power $P$.

${ }^{5}$ To ensure that the decimated channel observed through the pilot transmissions has an un-aliased spectrum, it is necessary that $\frac{T_{t}(s)}{T}>2 f_{D}$.
}

have an exponential distribution with mean $\sigma_{\widetilde{\mathbf{h}}}^{2}(s), 1-\sigma_{\widetilde{\mathbf{h}}}^{2}(s)$, respectively ${ }^{6}$ [5], [8], [13].

\section{THROUGHPUT MAXIMIZATION}

In this section, we focus on our main goal: the optimization of pilot overhead in the case of ARQ feedback. Our analysis is valid under the following idealized assumptions: identical packet sizes, capacity achieving codes, negligible signaling overhead, perfect feedback and fixed rate target $R$ in bits/Hz/s. To obtain the throughput we first need an expression for average rate per transmission. This depends on the the probability of outage, or that the target rate we are transmitting at, $R$, is above what the channel may support, modeled as its mutual information. We define the event $A_{s}:=\left\{I_{s}>R\right\}$, where $I_{s}$ is the mutual information (MI) after the $s$-th transmission/packet. Under Gaussian inputs and noise, the MIs are functions of the Signal to Interference plus Noise Ratio (SINR) $\beta(s)$ as

$$
I_{s}=(1-\alpha(s)) \log (1+\beta(s)),
$$

where $\beta(s)=\frac{\left|\widehat{\mathbf{h}}_{s}\right|^{2} P}{N_{o}+\left|\widetilde{\mathbf{h}}_{s}\right|^{2} P}$ and $\alpha(s)=\frac{T_{t}(s)}{T}$. The mismatch $\widetilde{\mathbf{h}}_{s}=\mathbf{h}_{s}-\widehat{\mathbf{h}}_{s}$ is unknown at the destination, and hence considered unresolvable.

\section{A. Derivation of Outage Probability}

We state the probability $p(m)$ that the random sequence $I_{1}, I_{2}, \ldots, I_{m}$ of mutual information at the user decoder did not cross the level $R$ at the $m$-th step, $p(m)=$ $\mathrm{P}_{r}\left\{\overline{A_{1}}, \overline{A_{2}}, \ldots, \overline{A_{m}}\right\}$. The $p(m)$ for ARQ is derived as follows [1]:

$$
\begin{aligned}
p(m) & =\prod_{s=1}^{m} \operatorname{Pr}((1-\alpha(s)) \log (1+\beta(s))<R) \\
& =\prod_{s=1}^{m} \operatorname{Pr}\left(\left|\hat{\mathbf{h}}_{s}\right|^{2}<\frac{N_{0} \gamma(s)}{P}+\gamma_{s}\left|\tilde{\mathbf{h}}_{s}\right|^{2}\right) \\
& :=\prod_{s=1}^{m} P_{o}(s)
\end{aligned}
$$

where $P_{o}(s)=\left(1-\frac{1-\sigma_{\tilde{\mathbf{h}}}^{2}(s)}{1-\sigma_{\tilde{\mathbf{h}}}^{2}(s)+\gamma(s) \sigma_{\tilde{\mathbf{h}}}^{2}(s)} \exp \left(-\frac{N_{0} \gamma(s)}{P-P \sigma_{\tilde{\mathbf{h}}}^{2}(s)}\right)\right)$ and $\gamma(s)=\exp \left(\frac{R}{1-\alpha(s)}\right)-1$.

\section{B. Maximization of Throughput}

We define the throughput as $\nu=\frac{\mathcal{R}}{\mathcal{T}}$ where, assuming maximum $M$ transmissions ${ }^{7}$, the expected rate $\mathcal{R}$ in bits $/ \mathrm{Hz} / \mathrm{s}$ is $\mathcal{R}=R(1-p(M))$, and the expected number of transmission per packet $\mathcal{T}$ is $\mathcal{T}=1+\sum_{m=1}^{M-1} p(m)$. Our definition of throughput assumes all nodes always have packets to send [15], [16]. The goal of this research is to maximize the throughput with respect to $[\alpha(1), \ldots, \alpha(M)]$. Assuming ARQ re-transmission protocol and rectangular Doppler-spectrum

\footnotetext{
${ }^{6}$ Note that the distribution of $\widehat{\mathbf{h}}_{s}$ and $\widetilde{\mathbf{h}}_{s}$ vary with the resources allocated to training $T_{t}(s)$

${ }^{7}$ We stop transmitting the same packet after $M$ attempts.
} 
fading, the maximization of the throughput can be tackled as follows:

$$
\begin{aligned}
{\left[\alpha^{*}(1), \ldots, \alpha^{*}(M)\right]=} & \arg \max \frac{\mathcal{R}}{\mathcal{T}}, \\
& =\arg \max \frac{R(1-p(M))}{1+\sum_{m=1}^{M-1} p(m)}, \\
& =\arg \max \frac{R\left(1-\prod_{s=1}^{M} P_{o}(s)\right)}{1+\sum_{m=1}^{M-1} \prod_{s=1}^{m} P_{o}(s)}, \\
\Rightarrow \forall s=1, \ldots, M \quad & \alpha^{*}(s)=\arg \min P_{o}(s) .
\end{aligned}
$$

Equation (3) follows from the derivation of the throughput with respect to $[\alpha(1), \ldots, \alpha(M)]$ as:

$\forall s=1, \ldots, M \quad \frac{\partial \frac{\mathcal{R}}{\mathcal{T}}}{\partial \alpha(s)}=\frac{\partial P_{o}(s)}{\partial \alpha(s)} \frac{\left(-\mathcal{R} R-\mathcal{T} \sum_{m=1}^{M-1} \prod_{l=1, l \neq s}^{m} P_{o}(l)\right)}{\mathcal{T}^{2}}$

replacing $\exp \left(\frac{R}{1-x}\right)$ and $\exp \left(\frac{2 R}{1-x}\right)$ by their two-term power series expansions, we get the following quartic polynomial:

$$
\begin{aligned}
- & e^{R} \frac{P}{N_{o}} R\left(-2 f_{D}\left(1+\frac{P}{N_{o}}\right)+\frac{P}{N_{o}} R\right) x^{4} \\
& +\left(8 e^{R}\left(e^{R}-1\right) f_{D}^{2} R-e^{R}\left(\frac{P}{N_{o}}\right)^{2} R\right. \\
& -2 f_{D} \frac{P}{N_{o}}\left(1+\frac{P}{N_{o}}+2 e^{2 R} R^{2}\right. \\
& \left.\left.+e^{R}\left(2 R-1+\frac{P}{N_{o}}\left(R^{2}+2 R-1\right)\right)\right)\right) x^{3} \\
& -2 f_{D}\left(\left(e^{R}-1\right) \frac{P}{N_{o}}\left(2+2 \frac{P}{N_{o}}+e^{R} R\right)+f_{D}(-2\right. \\
& \left.\left.-2 e^{R}\left(R^{2}+4 R-2\right)+e^{2 R}\left(4 R^{2}+8 R-2\right)\right)\right) x^{2} \\
& +2\left(e^{R}-1\right) f_{D}\left(\frac{P}{N_{o}}\left(1+\frac{P}{N_{o}}\right)\right. \\
& \left.+2 f_{D}\left(2+e^{R}(R-2)\right)\right) x \\
& +4 f_{D}^{2}-8 e^{R} f_{D}^{2}+4 e^{2 R} f_{D}^{2}=0 .
\end{aligned}
$$

In this case, the maximization of the throughput is reduced to the minimization of the outage probability $P_{o}(s), \forall s=$ $1, \ldots, M$. This minimization is performed independently at each transmission $s$. Note that Equation (3) is not valid if we use Hybrid-ARQ re-transmission protocols (e.g. chase combining or incremental redundancy). By differentiating $P_{o}(s)$, the optimization problem is distilled to solving the following equation for $x \in[0,1]$

$$
\begin{array}{r}
e^{\frac{R}{1-x}}\left(-8 f_{D}^{2}+16 f_{D}^{2} x+2 f_{D} \frac{P}{N_{o}} x+2 f_{D}\left(\frac{P}{N_{o}}\right)^{2} x\right. \\
+4 f_{D}^{2} R x-8 f_{D}^{2} x^{2}-4 f_{D} \frac{P}{N_{o}} x^{2}-4 f_{D}\left(\frac{P}{N_{o}}\right)^{2} x^{2} \\
\left.-2 f_{D}\left(\frac{P}{N_{o}}\right)^{2} R x^{2}+\left(2 f_{D} \frac{P}{N_{o}}+2 f_{D}\left(\frac{P}{N_{o}}\right)^{2}-\left(\frac{P}{N_{o}}\right)^{2} R\right) x^{3}\right) \\
+e^{\frac{2 R}{1-x}}\left(4 f_{D}^{2}-8 f_{D}^{2} x-4 f_{D}^{2} R x+4 f_{D}^{2} x^{2}-2 f_{D} \frac{P}{N_{o}} R x^{2}\right) \\
+4 f_{D}^{2}-8 f_{D}^{2} x-2 f_{D} \frac{P}{N_{o}} x-2 f_{D}\left(\frac{P}{N_{o}}\right)^{2} x+4 f_{D}^{2} x^{2} \\
+4 f_{D} \frac{P}{N_{o}} x^{2}+4 f_{D}\left(\frac{P}{N_{o}}\right)^{2} x^{2} \\
-2 f_{D} \frac{P}{N_{o}} x^{3}-2 f_{D}\left(\frac{P}{N_{o}}\right)^{2} x^{3}=0 .
\end{array}
$$

\section{Pilot overhead optimization}

Solving the previous equation does not yield an explicit solution and therefore must be computed numerically. Next, we overcome this difficulty by appropriately expanding the exponential function. In particular, we will expand it with respect to $x \approx 0$. This leads to a quartic polynomial that can be solved analytically. In addition to being analytically convenient, this approach illustrates the dependency of $x$ on the parameters of interest such as $R, f_{D}$, and $\frac{P}{N_{o}}$. After
This quartic polynomial has real coefficients and can be factored. The factoring procedure is outlined in detail in [17]. It is briefly summarized in the appendix ${ }^{8}$. We discard the roots that are not in the interval $[0,1]$. The roots of the Equation (4) are shown alongside the exact numerical value in Fig. 2, for $R=1,2$ and 3 and $f_{D}=0.5,0.2$ and 0.05 . By examining the polynomial coefficients in Equation (4) we notice that when $f_{D} \approx 0$ all but the high-order polynomial coefficient $\approx 0$, hence the optimal pilot overhead is very small $(x \approx 0)$.

High-power regime: For high-power regime, assuming that $\left(\frac{P}{N_{o}}\right)^{2} \gg \frac{P}{N_{o}} \gg R$ yields to the simplification of Equation (4) as follow:

$$
\begin{aligned}
& \frac{-e^{R} R\left(R-2 f_{D}\right)}{2 f_{D}\left(e^{R}-1\right)} x^{3} \\
& \quad-\frac{\left(2 f_{D}\left(1+e^{R}\left(R^{2}+2 R-1\right)\right)+e^{R} R\right)}{2 f_{D}\left(e^{R}-1\right)} x^{2} \\
& \quad-2 x+1=0 .
\end{aligned}
$$

Note that the roots of this polynomial are not function of the power, which is validated by the exact numerical values in Fig. 2. The optimization of the pilot overheard is then reduced to solving a simple cubic polynomial. By examining the polynomial coefficients ${ }^{9}$, we prove that the optimal pilot overhead decrease with $R$ and increase with $f_{D}$.

Low-power regime: For low-power regime, assuming that $\left(\frac{P}{N_{o}}\right)^{2} \ll \frac{P}{N_{o}} \ll R$ yields to the simplification of Equation (4) as follow:

$$
\begin{aligned}
& 2 e^{R}\left(e^{R}-1\right) R x^{3} \\
& \quad-\left(e^{2 R}\left(2 R^{2}+4 R-1\right)-e^{R}\left(R^{2}+4 R-2\right)-2\right) x^{2} \\
& \quad+ \\
& \quad\left(e^{R}-1\right)\left(2+e^{R}(R-2)\right) x+1-2 e^{R}+e^{2 R}=0 .
\end{aligned}
$$

${ }^{8}$ For clarity purpose, we decided here to display the polynomial instead of the roots.

${ }^{9}$ The two high-order polynomial coefficients increase with $R$ and decrease with $f_{D}$. 

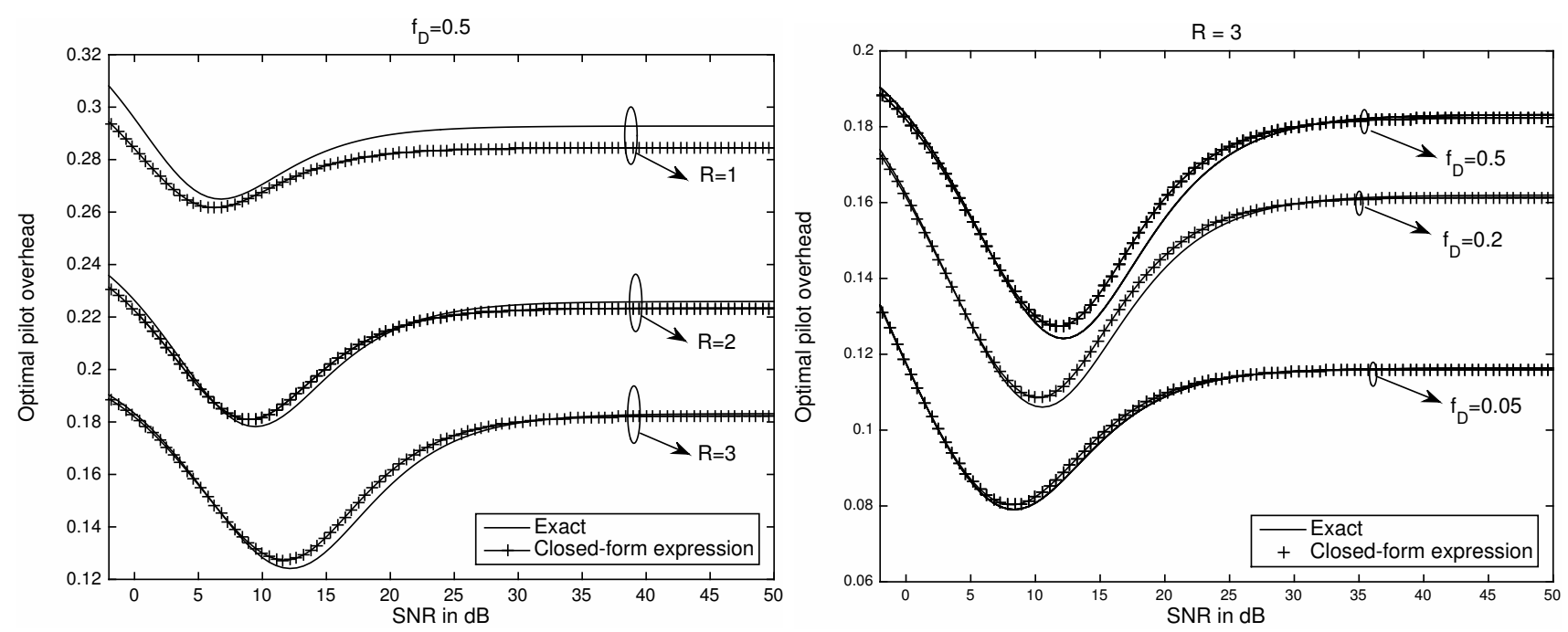

Fig. 2: Optimal pilot overhead.

Note that the roots of this polynomial are only function of $R$, as validated by Fig. 2 .

\section{REFERENCES}

[1] B. Smida, N. Devroye, and T. Li, "Optimization of two-way communication with ARQ feedback," in Proc. IEEE Int. Conf. Commun. IEEE, 2013.

[2] J. Cavers, "An analysis of pilot symbol assisted modulation for rayleigh fading channels [mobile radio]," Vehicular Technology, IEEE Transactions on, vol. 40, no. 4, pp. 686-693, Nov 1991.

[3] M. Medard, "The effect upon channel capacity in wireless communications of perfect and imperfect knowledge of the channel," Information Theory, IEEE Transactions on, vol. 46, no. 3, pp. 933-946, May 2000.

[4] J. Baltersee, G. Fock, and H. Meyr, "An information theoretic foundation of synchronized detection," Communications, IEEE Transactions on, vol. 49, no. 12, pp. 2115-2123, Dec 2001.

[5] B. Hassibi and B. Hochwald, "How much training is needed in multipleantenna wireless links?" IEEE Trans. Inf. Theory, vol. 49, no. 4, pp. 951 $-963,2003$.

[6] L. Tong, B. Sadler, and M. Dong, "Pilot-assisted wireless transmissions: general model, design criteria, and signal processing," "IEEE Signal Proc. Mag.”, vol. 21, no. 4, pp. 12 - 25, 2004.

[7] S. Ohno and G. Giannakis, "Average-rate optimal psam transmissions over time-selective fading channels," IEEE Trans. Wireless Comm., vol. 1, no. 4, pp. $712-720,2002$.

[8] H. Vikalo, B. Hassibi, B. Hochwald, and T. Kailath, "Optimal training for frequency-selective fading channels," in Proc. IEEE Int. Conf. Acoust., Speech, Signal Process. IEEE, 2001, pp. 2105 - 2108.

[9] X. Ma, G. B. Giannakis, and S. Ohno, "Optimal training for block transmissions over doubly selective wireless fading channels ," IEEE Trans. Signal Processing, vol. 51, no. 5, pp. 1351-1366, 2003.

[10] M. Dong, L. Tong, and B. Sadler, "Optimal insertion of pilot symbols for transmissions over time-varying flat fading channels," Signal Processing, IEEE Transactions on, vol. 52, no. 5, pp. 1403-1418, May 2004.

[11] S. Furrer and D. Dahlhaus, "Multiple-antenna signaling over fading channels with estimated channel state information: Capacity analysis," Information Theory, IEEE Transactions on, vol. 53, no. 6, pp. 20282043, June 2007.

[12] A. Lozano, "Interplay of spectral efficiency, power and doppler spectrum for reference-signal-assisted wireless communication," Wireless Communications, IEEE Transactions on, vol. 7, no. 12, pp. 5020-5029, December 2008

[13] N. Jindal and A. Lozano, "A unified treatment of optimum pilot overhead in multipath fading channels," Communications, IEEE Transactions on, vol. 58, no. 10, pp. 2939-2948, October 2010.

[14] C. Jakes, Microwave mobile communications. John Wiley and sons, 1974.

[15] G. Caire and D. Tuninetti, "The throughput of hybrid-ARQ protocols for the gaussian collision channel," IEEE Trans. Inf. Theory, vol. 47, no. 5 , pp. $1971-1988$, July 2001 .
[16] P. Larsson, B. Smida, T. Koike-Akino, and V. Tarokh, "Analysis of network coded HARQ for multiple unicast flows," Communications, IEEE Transactions on, vol. 61, no. 2, pp. 722-732, February 2013.

[17] E. L. Rees, "Graphical discussion of the roots of a quartic equation," The American Mathematical Monthly, vol. 29, no. 2, pp. pp. 51-55, 1922. [Online]. Available: http://www.jstor.org/stable/2972804

\section{APPENDIX}

Assuming a quartic polynomial $a x^{4}+b x^{3}+c x^{2}+d x+e$. The roots are given by

$$
\begin{aligned}
& x_{1}=-\frac{b}{4 a}-S+\frac{1}{2} \sqrt{-4 S^{2}-2 p+\frac{q}{S}}, \\
& x_{2}=-\frac{b}{4 a}-S-\frac{1}{2} \sqrt{-4 S^{2}-2 p+\frac{q}{S}}, \\
& x_{3}=-\frac{b}{4 a}+S+\frac{1}{2} \sqrt{-4 S^{2}-2 p+\frac{q}{S}}, \\
& x_{4}=-\frac{b}{4 a}+S-\frac{1}{2} \sqrt{-4 S^{2}-2 p+\frac{q}{S}} .
\end{aligned}
$$

Where, the discriminant is $\Delta=256 a^{3} e^{3}-192 a^{2} b d e^{2}-$ $128 a^{2} c^{2} e^{2}-27 a^{2} d^{4}+144 a b^{2} c e^{2}-6 a b^{2} d^{2} e-80 a b c^{2} d e+$ $18 a b c d^{3}+16 a c^{4} e-4 a c^{3} d 2-27 b^{4} e^{2}+18 b^{3} c d e-4 b^{3} d^{3}-$ $4 b^{2} c^{3} e+b^{2} c^{2} d^{2}$, and the following intermediate variables are $\Delta_{0}=c^{2}-3 b d+12 a e, \Delta_{1}=\sqrt{4 \Delta_{0}^{3}-27 \Delta}, p=$ $\frac{8 a c-3 b^{2}}{8 a^{2}}, q=\frac{b^{3}-4 a b c+8 a^{2} d}{8 a^{3}} Q=\sqrt[3]{\frac{\Delta_{1}+\sqrt{-27 \Delta}}{2}}$, and $S=$ $\frac{1}{2} \sqrt{\frac{2}{3} p+\frac{1}{3 a}\left(Q+\frac{\Delta_{0}}{Q}\right)}$. 